\title{
The APOKASC Catalog
}

Jennifer A. Johnson

\begin{abstract}
I report on the APOKASC catalog, a joint effort between the Kepler Asteroseismic Science Consortium and the SDSS-III APOGEE spectroscopic survey. It will contain both seismic and spectroscopic values for stars observed by both surveys. I discuss the derivation of spectroscopic parameters and their uncertainties from the H-band spectra delivered by the APOGEE spectrograph, illustrating the sensitivity of stellar spectra to some parameters, such as $T_{\text {eff }}$, and lack of sensitivity to others, such as logg.
\end{abstract}

\section{Contributors to the APOKASC catalog}

This catalog is the result of intensive effort by KASC and APOGEE scientists, particularly those who have been working on the analysis of Kepler lightcurve data, grid-based modelling, and the APOGEE spectroscopic parameters pipeline (ASPCAP), but also the broader teams from the instrument builders to lead scientists. I acknowledge here in reverse alphabetical order the former group: Gail Zasowski, Nicholas Troup, Dennis Stello, Verne Smith, Victor Silva Aguirre, Matthew Shetrone, Aldo Serenelli, Ricardo Schiavon, Marc Pinsonneault, Benoit Mosser, Andrea Miglio, Travis Metcalfe, Szabolcs Mészáros, Savita Mathur, Steven R. Majewski, Daniel Huber, Jon Holtzman, Saskia Hekker, Leo Girardi, Rafael Garcia, Ana García Pérez, Courtney R. Epstein, Yvonne Elsworth, Katia Cunha, William Chaplin, Dmitry Bizyaev, Sarbani Basu, Carlos Allende Prieto.

Jennifer A. Johnson

Department of Astronomy, Ohio State University, 140 W. 18th Ave, Columbus, OH, 43210 e-mail:

jajeastronomy.ohio-state.edu 


\section{Overview of APOKASC Catalog}

The APOKASC catalog is a joint effort between the Kepler Asteroseismic Science Consortium and the SDSS-III APOGEE spectroscopic survey. For stars observed by both groups, the catalog will have the seismic parameters $\Delta v$ and $v_{\max }$, the spectroscopic parameters $T_{\text {eff }}$ metallicity and their uncertainties. In addition, grid-based modeling values for logg, mass and radius, derived using the combination of seismic and spectroscopic parameters, will be reported. The first version of the catalog will contain the results for $\sim 1900$ red giant stars (Pinsonneault, 2014); subsequent versions of the catalog will include thousands of additional red giants observed in Years 2-3 of APOGEE observations as well as hundreds of dwarf stars.

\section{Advantages of Combining Spectroscopic and Seismic Data}

Measurements of the fundamental properties of stars, such as masses, radii, ages, rotation profiles, evolutionary state, and composition, are crucial for understanding numerous issues in stellar structure and evolution, star formation histories, and stellar populations. Seismic measurements and spectroscopic measurement are very complementary in which characteristics they can measure. For example, logg is very well determined from the seismic parameters, while detailed composition measurements, such as $[\mathrm{Mg} / \mathrm{Fe}]$ or $[\mathrm{Na} / \mathrm{Fe}]$, will remain the purview of spectroscopy. Also, the addition of $\mathrm{T}_{\text {eff }}$ and metallicity information can improve the accuracy of gridbased modelling results for properties such as mass (e.g., Gai et al. 2011).

With this catalog, we plan to study a variety of areas, including the projects below, which have already been started from the preliminary version of the APOKASC catalog.

- Dependence of seismic properties on metallicity

- Surface and internal stellar rotation rates

- New identifications of stellar populations, e.g., merger remnants

- Improved distance measurements

- Tests of Galactic stellar population models from masses and radii of thousands of red giants

\section{Spectroscopic Data}

I focus this proceeding on describing the APOGEE spectroscopic observations and the sensitivity of near-IR spectra to stellar parameters, as this audience is most familar with the derivation and use of seismic parameters.

Page: 2 job:jaj_sesto macro:svmult.cls date/time:1-Oct-2018/5:48 


\subsection{Overview of APOGEE survey}

The Sloan Digital Sky Survey (SDSS) III (Eisenstein et al. 2011) uses a 2.5-meter telescope with a wide field of view (7 square degrees) at Apache Point Observatory (APO) to survey the sky. The APO Galactic Evolution Experiment (APOGEE) uses a fiber-fed multiobject near-infrared spectrograph to observe 230 science targets at a time (Majewski et al., 2010). An additional 35 fibers are reserved for observing hot stars to facilitate removal of telluric lines and 35 fibers are placed on regions of black sky. The spectra are high resolution $(\mathrm{R} \sim 22,500)$, high signal-to-noise $(\sim$ 100 per dithered resolution element) and cover the wavelength range of 1.51 to $1.68 \mu \mathrm{m}$. APOGEE began routine observations in Fall 2012. The first data release of APOGEE data, including the spectra for the stars that are part of the first APOKASC catalog, was the SDSS Data Release 10 (Ahn et al., 2014).

\subsection{Derivation of Stellar Parameters from Spectra}

The strengths of absorption features in the spectra of cool giants are sensitive to several properties of the atmosphere, including $\mathrm{T}_{\text {eff }}$ and $\operatorname{logg}$, which affect the excitation and ionization balance of the electrons and the molecular equilibrium, and compositon, which affects both the optical depth at the wavelengths of transitions and the continuous opacity through electron donation to form $\mathrm{H}^{-}$.

To derive the stellar atomspheric parameters from spectra, first, the raw data are processed through the data reduction pipeline to produce combined, wavelengthcalibrated, and radial-velocity-corrected spectra (Nidever, 2014). Next, stellar parameters are determined using ASPCAP (Garcia Perez, in prep), which finds the $\chi^{2}$ minimum difference between the observed spectra and a 6-D grid of synthetic spectra. The parameters that are varied to create the synthetic spectra are $\mathrm{T}_{\text {eff }}, \operatorname{logg}$, $[\mathrm{M} / \mathrm{H}],[\mathrm{C} / \mathrm{M}],[\mathrm{N} / \mathrm{M}]$ and $[\alpha / \mathrm{M}]$. To calculate spectra with different $[\mathrm{M} / \mathrm{H}]$, the abundances of all elements except $\mathrm{C}, \mathrm{N}$, and the $\alpha$ elements are changed up or down in the same proportion from their solar ratios. In as much as lines of Fe are the most common out of all the elements that comprise "M", the best-fit $[\mathrm{M} / \mathrm{H}]$ is an excellent proxy for $[\mathrm{Fe} / \mathrm{H}]$, a fact confirmed by comparison with cluster stars (Mészáros et al., 2013). Fitting to a 6-D grid is necessary because the depth of an absorption line is never sensitive to just one parameter. A critical assesment of the systematic and random uncertainties in the ASPCAP stellar parameters was performed by comparison with parameters for stars in well-studied globular and open clusters and for stars in the "gold standard" KASC seismic sample in the Kepler field (Mészáros et al., 2013).

Page: 3 job:jaj_sesto macro:svmult.cls date/time:1-Oct-2018/5:48 


\subsubsection{Effective Temperature}

The stellar parameter that has the most effect on the appearance of a stellar spectrum is the $\mathrm{T}_{\text {eff }}$. Fig 1 shows two stars in the APOKASC catalog whose temperatures differ by a mere $20 \%$, yet the strength of the Brackett lines varies markedly. There are numerous lines that appear in the cooler star that are weak or non-existent in the hotter star as either the excitation or the ionization state is not appropriate for absorption of near-IR photons. The strong dependence on temperature in the Boltzmann and Saha equations and the presence of numerous lines of minority species, such as neutral atoms and molecules, means that small temperature uncertainties translate into larger uncertainties in $[\mathrm{M} / \mathrm{H}]$.

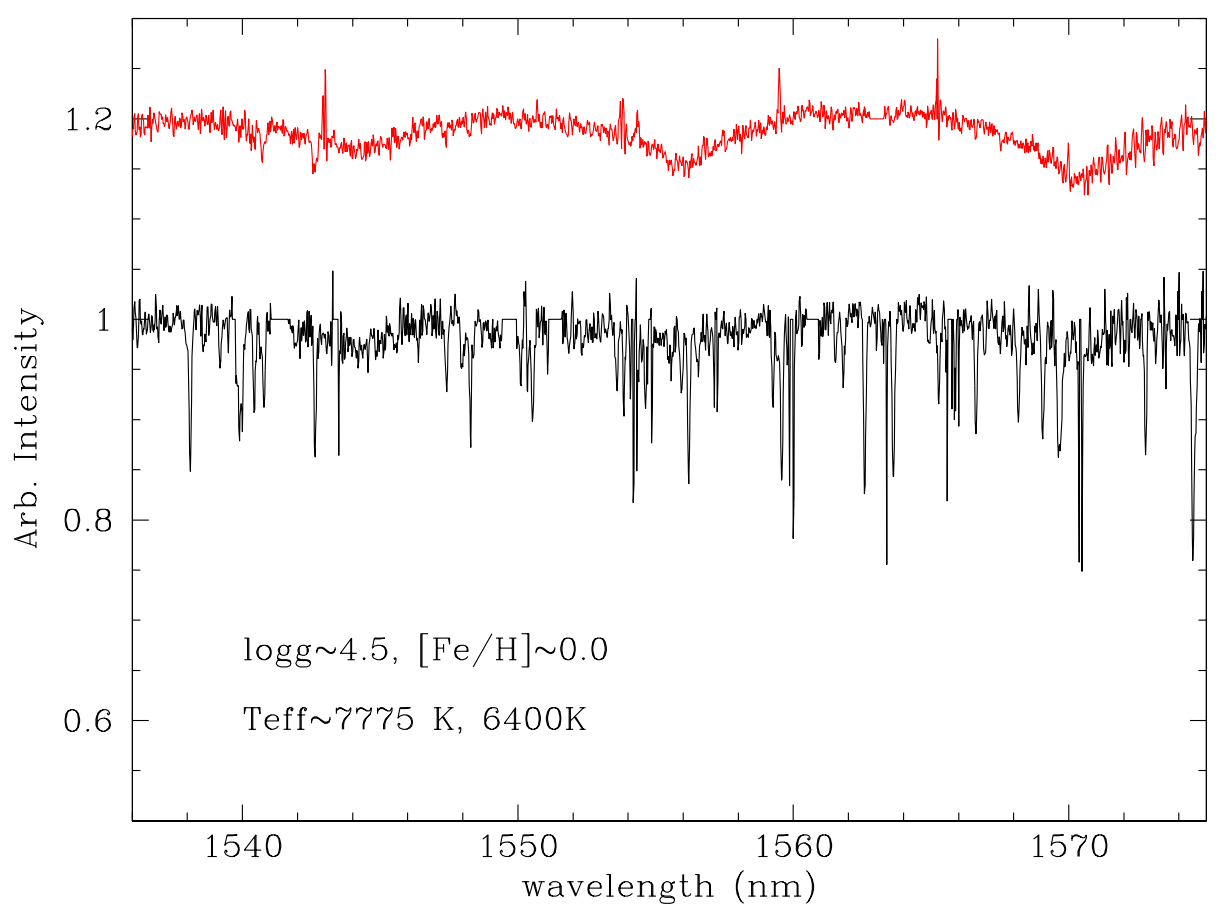

Fig. 1 This figure shows parts of the spectra of two stars in the APOKASC catalog that differ by $\sim 1400 \mathrm{~K}$ or $\sim 20 \%$ in Teff, but have similar $\operatorname{logg}$ and $[\mathrm{M} / \mathrm{H}]$. Despite the relative small change in the temperature, there are large differences in the appearance between the hotter star (top) and the cooler star (bottom).

To determine the accuracy of the ASPCAP-derived $\mathrm{T}_{\text {eff }}$, we compared the $\mathrm{T}_{\text {eff }}$ from the spectroscopic pipeline to photometric temperatures derived from the IRFM-based color- $\mathrm{T}_{\text {eff }}$ for 2MASS filters González Hernández \& Bonifacio (2009) for cluster stars with well-determined reddenings. The ASPCAP temperatures were

Page: 4 job: jajsesto macro: svmult.cls date/time:1-Oct-2018/5:48 
$\sim 150 \mathrm{~K}$ lower than the photometric ones on average, with a clear trend with temperature. A correction was applied to the ASPCAP temperatures to bring them into better agreement with the latter values. After the correction, the scatter in difference between the two measurements was $<200 \mathrm{~K}$, dropping to $<100 \mathrm{~K}$ for stars with solar-like metallicity or higher.

The advantage of determing a systematic correction to the ASPCAP temperatures, rather than using photometric temperatures for all APOGEE stars, is that a correctly calibrated spectroscopic temperature is reliable even if the reddening is uncertain. Many APOGEE fields lie in the midplane of the Galactic disk in regions of highly variable extinction, and the reddening map in the Kepler field itself is currently being revised (Zasowski, 2014; Huber et al., 2014).

\subsubsection{Metallicity}

As Fig 2] shows, the composition of a star has an obvious effect on the appearance of a spectrum, as more metal-rich stars show much deeper absorption lines than a star with many fewer heavy-element atoms in its atmosphere. The problem is, of course, that temperature in particular has a much larger effect. The stars in Fig 1 differ in $\mathrm{T}_{\text {eff }}$ by $\sim 20 \%$, while the two stars in Fig 2 differ in metal content by a factor of 100 ! Therefore, the uncertainties in metallicity will be much larger than the $4 \%$ errors in $\mathrm{T}_{\text {eff }}$ discussed above.

The cluster stars provide the crucial test of the uncertainties and systematic offsets for the ASPCAP metallicity measurements, as all cluster stars should have similar metallicity, at least at the level of precision discussed here (e.g., Carretta et al. 2009), although subsequent analysis will take into account the variation in globular clusters of the light elements (e.g., Kraft 1994). We compared the [M/H] derived by ASPCAP to the $[\mathrm{Fe} / \mathrm{H}]$ values reported in the literature for clusters spanning a metallicity range of $-2.3<[\mathrm{Fe} / \mathrm{H}]<+0.3$ (Mészáros et al., 2013). In general, there was quite good agreement, confirming that the $[\mathrm{M} / \mathrm{H}]$ value is a good proxy for $[\mathrm{Fe} / \mathrm{H}]$. For metal-rich clusters, the initial ASPCAP values are only about 0.1 too high, while they are 0.35 dex for metal-poor clusters. A metallicity-dependent correction is applied to the $[\mathrm{M} / \mathrm{H}]$ values. The uncertainity in $[\mathrm{M} / \mathrm{H}]$, determined by looking at the scatter in the corrected values compared to literature values, ranges from 0.15 dex for the most metal-poor clusters to $\sim 0.05$ dex for clusters with solar metallicity and above. The strong metallicity dependence in the uncertainty in metallicity is not surprisingly, as metal-poor stars have noticeably fewer metal lines to use for the measurement.

To assess the difference that the corrected and uncorrected temperature scales and metallicity scales have on the results of the grid-based modelling, two separate computations were done. Uncorrected $[\mathrm{M} / \mathrm{H}]$ were used in combination with uncorrected $\mathrm{T}_{\text {eff }}$ values in one case, while corrected values are used in the second case. Two sets of logg, masses and radii are reported.

Page: 5 job:jaj_sesto macro: svmult.cls date/time:1-oct-2018/5:48 


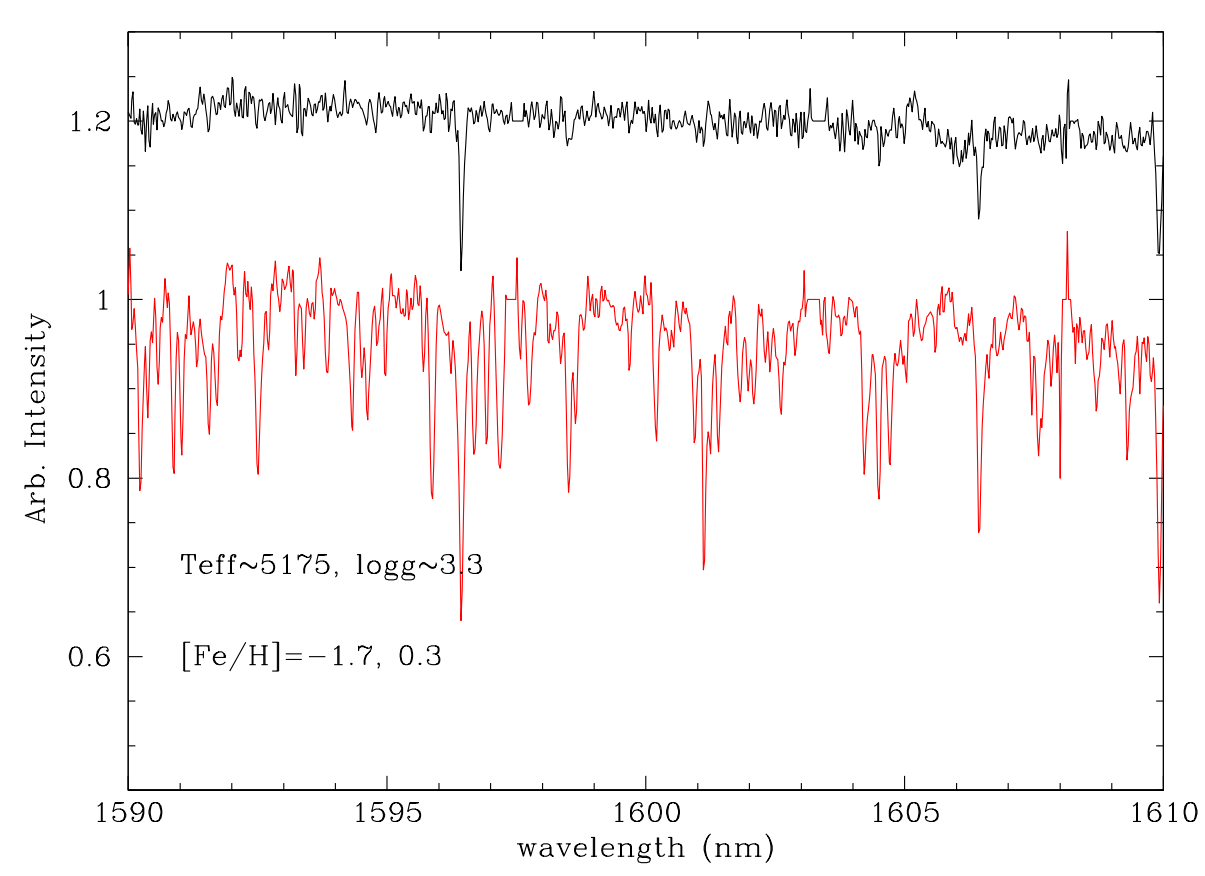

Fig. 2 This figure shows parts of the spectra of two stars in the the APOKASC catalog that differ by $\sim 2 \mathrm{dex}$ in $[\mathrm{M} / \mathrm{H}]$, but have similar $\mathrm{T}_{\text {eff }}$ and logg. The number of heavy atoms in the atmosphere has a clear effect on the spectrum, as the more metal-poor star's spectrum (top) has considerably fewer lines than the more metal-rich star's spectrum (bottom).

\subsubsection{Gravity}

Logg values, unlike temperature and metallicity, can be measured by both seismic and spectroscopic techniques. However, the spectroscopic gravity measurements have larger uncertainties than the seismic measurements because the spectra of stars in the H-band are not particularly sensitive to logg changes (see Fig 3). We compared the ASPCAP logg's with values both from seismic analysis of a few hundred stars in the Kepler field as well as with the logg's predicted for cluster stars from isochrones matched to the cluster distance and reddening (Mészáros et al., 2013). The values initially reported by the ASPCAP pipeline are too high compared to the seismic and isochrone stars by at least $0.2 \mathrm{dex}$, rising to $0.5 \mathrm{dex}$ for metal-poor stars. A correction is again applied, leading to much better agreement. A scatter of $\sim 0.15$ dex is present in the differences between corrected ASPCAP and calibration values, providing an estimate of the remaining uncertainty in the spectroscopic values. Part of that scatter is because it was difficult to reconcile (at the $\sim 0.1$ dex level) the offsets between the seismic values in the Kepler field and the values determined from

Page: 6 job:jaj_sesto macro:svmult.cls date/time:1-Oct-2018/5:48 
isochrones for stars with similar $\mathrm{T}_{\text {eff }}$ and gravity in the clusters. This difference is particularly striking for the metal-rich stars and remains under investigation by the ASPCAP team.

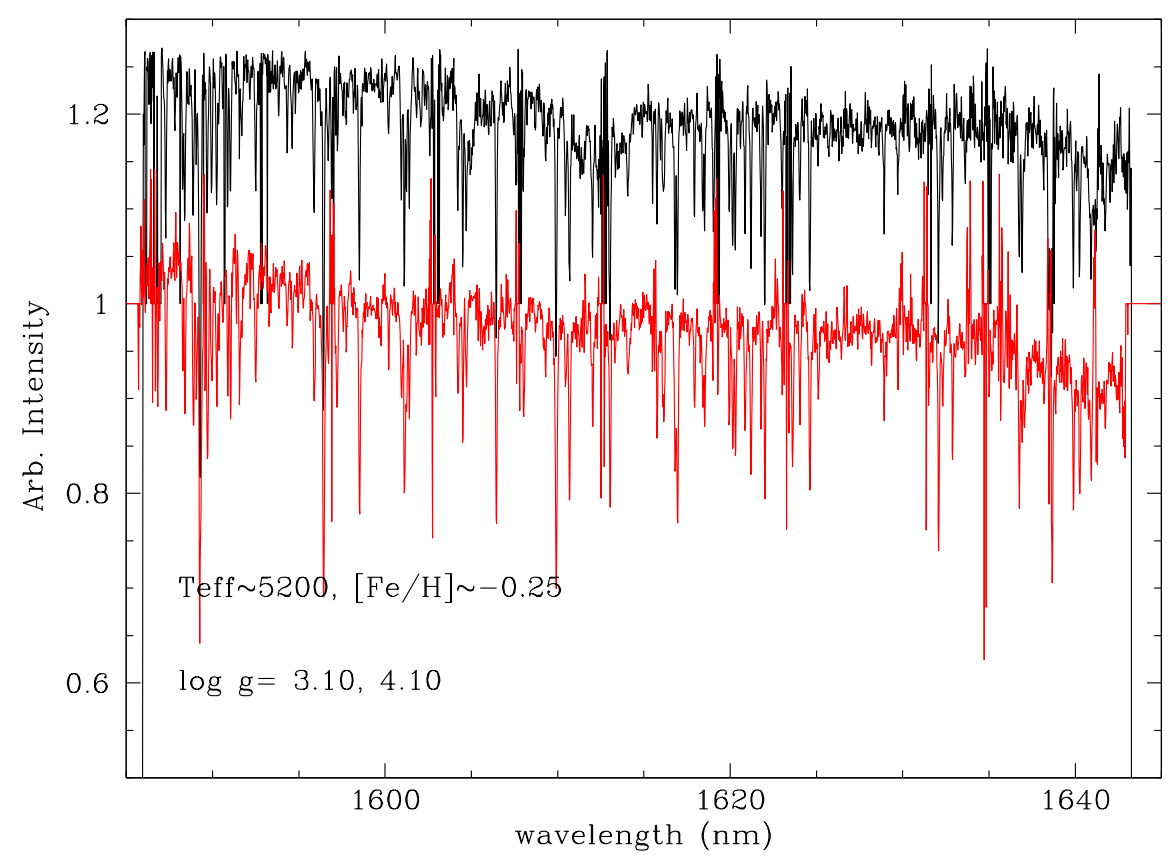

Fig. 3 This figure shows parts of the spectra of two stars in the APOKASC catalog that differ by $\sim 1$ dex in logg, but have similar Teff and $[\mathrm{M} / \mathrm{H}]$. The differences between the lower gravity star (top) and higher gravity star (bottom) are not as obvious as for 1 and 2 demonstrating why the uncertainties in gravity are large for spectroscopically determined values.

\section{Conclusion}

In this proceeding, I have summarized the APOGEE data and the derivation of stellar parameters and their uncertainties from high-resolution H-band data. These parameters, along with the seismic parameters $\Delta v$ and $v_{\max }$ are included in the APOKASC catalog as well as being passed to grid-based modellers for logg, mass, and radii determinations that are also included in the catalog. The publication of this catalog will, I hope, aid research in studies of stellar structure, stellar atmospheres, and stellar populations.

Page: 7 job:jaj_sesto macro:svmult.cls date/time:1-Oct-2018/5:48 
Acknowledgements A special thank you to Courtney Epstein, for her tireless work on targeting seismic sources in APOGEE and on gathering all the data into the catalog. And thank you to everyone who measured parameters for thousands of stars, no small task!

\section{References}

Ahn, C. P., Alexandroff, R., Allende Prieto, C., et al. 2014, ApJS , 211, 17

Carretta, E., Bragaglia, A., Gratton, R., D’Orazi, V., \& Lucatello, S. 2009, A\&A, 508, 695

Eisenstein, D. J., Weinberg, D. H., Agol, E., et al. 2011, AJ, 142, 72

Gai, N., Basu, S., Chaplin, W. J., \& Elsworth, Y. 2011, ApJ , 730, 63

Garcia Perez, A. e. a. in prep, in preparation,

González Hernández, J. I. \& Bonifacio, P. 2009, A\&A, 497, 497

Huber, D., Silva Aguirre, V., Matthews, J. M., et al. 2014, ApJS , 211, 2

Kraft, R. P. 1994, PASP, 106, 553

Majewski, S. R., Wilson, J. C., Hearty, F., Schiavon, R. R., \& Skrutskie, M. F. 2010, in IAU

Symposium, Vol. 265, IAU Symposium, ed. K. Cunha, M. Spite, \& B. Barbuy, 480-481

Mészáros, S., Holtzman, J., García Pérez, A. E., et al. 2013, AJ, 146, 133

Nidever, D. e. a. 2014, in preparation

Pinsonneault, M. e. a. 2014, in preparation

Zasowski, G. e. a. 2014, in preparation 